\title{
Chemotherapeutic Resistance in Egyptian Acute Myeloid Leukemia Patients
}

\author{
Neemat M Kassem ${ }^{1}$, Nashwa Medhat ${ }^{2}$, Hebatallah A Kassem ${ }^{1 *}$, Mohamed A \\ El-Desouky ${ }^{3}$
}

\begin{abstract}
Background: Acute Myeloid Leukemia (AML) is a heterogeneous disorder with variable genetic abnormalities and cytogenetic alterations which provide a significant disease prognosis and determine response to therapy. Purpose: We aim to investigate the expression of the MDR1 gene in 100 Egyptian AML patients, to identify their role on both the progression and chemotherapeutic refractoriness together with assessment of known prognostic molecular markers; FLT3-ITD and NPM1 mutations. Methodology: Quantitative assessment of MDR1 gene expression was performed by quantitative RT-PCR. Additional prognostic molecular markers were determined as internal tandem duplications of the FLT 3 gene and nucleophosmin gene mutation A. Results: MDR1 gene expression levels and FLT3/ITD mutations were significantly higher in AML patients with resistant disease with $\mathrm{P}$ value $<0.001$ and 0.002 respectively. However, NPM1 was insignificantly higher in patients with CR P-value 0.14 . In MDR positive group, wild FLT3/ITD with or without NPM1 mutation was favorable in achieving CR with $\mathrm{p}$ value 0.02 . MDR negative group, wild FLT3/ITD with or without NPM1 mutation showed insignificantly higher CR rates with $\mathrm{p}$ value (0.35). Kaplan-Meier curves revealed statistically significant difference between MDR1-negative and MDR1-positive patients regarding their DFS and OS between the two groups where DFS and OS were higher in MDR1-negative patients with $p$ value 0.004 and 0.01 , respectively. Conclusion: The results obtained by the current work together with the previous researches concerning the study of multidrug resistance genes in AML patients provide additional evidence of the role played by these genes as predictors of chemoresistance and poor treatment outcome.
\end{abstract}

Keywords: Acute myeloid leukemia- chemoresistance- treatment outcome

Asian Pac J Cancer Prev, 20 (8), 2421-2427

\section{Introduction}

Acute Myeloid Leukemia (AML) is a heterogeneous disorder with variable genetic abnormalities and cytogenetic alterations which provide a significant disease prognosis and determine response to therapy (Liu et al., 2007). Specific AML entities had been identified by WHO through focusing on significant cytogenetic and molecular genetic subgroups. In the entity of AML with recurrent genetic abnormalities, the most commonly identified prognostic balanced cytogenetic abnormalities are $\mathrm{t}(8 ; 21)(\mathrm{q} 22 ; \mathrm{q} 22)$ and $\operatorname{inv}(16)(\mathrm{p} 13.1 \mathrm{q} 22)$. They are usually associated with a good response to chemotherapy and a high complete remission (CR) rate with long term disease-free survival (DFS). Therefore, considerable progress has been made not only in understanding AML pathogenesis, but also in the development of diagnostic assays and novel therapies (Döhner et al., 2015). In the last decade, many somatically acquired mutations have been identified in several genes in cytogenetically normal
(CN) AML such as FLT3-ITD, NPM1, CEBPA ...etc. The fms-related tyrosine kinase 3 (FLT3) gene is a member of type III receptor tyrosine kinase (RTK) and located on chromosome 13q12 (Grafone et al., 2012). Mutations in FLT3 predict inferior response to chemotherapy and poor overall survival in AML patients. FLT3 mutations have been found in $\sim 30 \%$ to $35 \%$ of adult AML patients. These mutations have been preferentially found in AML patients with normal karyotype (Takahashi et al., 2011). Mutations that result in the constitutive activation of FLT3 have been identified in two functional domains of the receptor. Internal tandem duplications (ITDs) in the juxtamembrane domain (JM) and activating point mutations in the second tyrosine kinase domain (TKD) which occur in $\sim 30 \%$ and $10 \%$ of AML patients, respectively (Leung et al., 2013). FLT3 mutations causes activation of signal transduction networks mainly PI3K-AKT, RAS-MEK-MAPK, and STAT5 pathways and result in uncontrolled cell proliferation. Also, these mutations suppress myeloid transcription factors 
PU.1, C/EBP $\alpha$, which result in blocking of myeloid differentiation. Therefore, FLT3 mutations have a crucial role in AML pathogenesis, and FLT3 inhibitors such as Midostaurin (PKC412, Novartis) have been validated by FDA as promising targeted therapy in combination with chemotherapy which used in newly diagnosed FLT3-ITD positive AML cases (Wei et al., 2017). The nucleophosmin 1 (NPM1) gene is located on chromosome 5q35 and contains 12 exons. It is an abundant phosphoprotein in nucleoli. It is primarily localized to nucleolus but has been shown to shuttle between the cytoplasm and nucleus. NPM1 mutations results in the cytoplasmic dislocation of NPM1 and that altered protein results in leukemogenesis. Prevalence of NPM1 mutations increases with age, occurring in $2-8 \%$ of childhood AML and $27 \%-35 \%$ of adult AML. NPM1 mutations occur in $45-64 \%$ of adult AML with a normal. This disease appears to show a female predominance (Suleiman et al., 2018). Regarding risk stratification of AML patients, AML with $\mathrm{t}(8 ; 21)$ (q22;q22.1); RUNX1-RUNX1T1 inv(16)(p13.1;q22) and mutated NPM without or with FLT3-ITD low shows a good response to induction chemotherapy with favorable outcome. Patients with mutated NPM1 and FLT3-ITD high or wild type NPM1 without FLT3-ITD or with FLT3-ITD low have intermediate risk stratification. The coexistence of wild-type NPM1and FLT3-ITD high is associated with an adverse prognosis and poor outcome (Daver et al., 2019). Multidrug resistance (MDR), the principal mechanism by which many cancers develop resistance to chemotherapy, is one of the major obstacles to the successful AML treatment (Hatakeyama and Harashima, 2014). Pediatric AML accounts for 15\%-20\% of all pediatric acute leukemias. Induction chemotherapy lead to complete remission (CR) in 50 to $90 \%$ of de novo patients, but 10 to $25 \%$ of patients have primary resistant disease and the most patients who gain remission relapses within 3 years of diagnosis (Styczynski, 2007). In adult AML patients, increasing age is independently associated with poorer outcomes. General health, performance status and different comorbidities have an important impact on the tolerance these patients have to intensive treatment modalities whereas specific age-related AML-associated genetic abnormalities increase the drug resistance. Hence, age should not be the sole determinant of treatment decisions (Almeida and Ramos, 2016) Expression of the multidrug resistance (MDR1) phenotype, encoded by the $M D R 1$ gene, is an adverse prognostic factor for CR and survival in acute myeloid leukemia (AML). The MDR1 gene encodes the drug transporter P glycoprotein (Pgp) which promotes drug efflux. The mechanism by which cancer cells resist to chemotherapy is the overexpression of Pgp (Scheffer et al., 1995). In AML patients, MDR1 gene expression was also associated with lower CR rates, but not decreased OS. Furthermore, analysis of MDR1 expression and FLT3-ITD mutation status in adult AML patients demonstrated shorter time to relapse in MDR1 overexpressing patients, and poor DFS in patients with both MDR1 overexpression and FLT3-ITD positive status (Tiribelli et al., 2011). This study aimed to investigate the impact of $M D R 1$ gene expression together with FLT3-ITD and NPM1 mutations on response to therapy in Egyptian
AML patients.

\section{Materials and Methods}

\section{Subject and methods \\ Study population}

The present study included 100 AML patients. Patients were recruited from the Kasr Al-Ainy Center of Clinical Oncology and nuclear medicine, School of Medicine, Cairo University. Twenty age and sex matched healthy volunteers were included in the current study as control group. For patients and controls, $2 \mathrm{ml}$ EDTA blood samples was collected under complete aseptic conditions for molecular studies. The study was approved by the Research ethical Committee of Clinical Oncology department, School of Medicine, Cairo University, and informed consents were obtained from all participants prior to enrollment in the study.

\section{RNA extraction}

Extraction of total RNA was performed by QIAamp RNA Blood Mini Kit (Qiagen, Germany) according to manufacturer's instructions. RNA integrity was tested on the Nanodrop (ND-1000) and stored at $-80{ }^{\circ} \mathrm{C}$. Total RNA was reverse transcribed using random primers with a high-capacity cDNA archive kit (Applied Biosystem, Foster city, CA, USA).

\section{Detection of FLT3/ITDs and NPM1 mutation A}

Briefly, the FLT3 gene was amplified using the following forward primer sequence: 5'CATTGTCGTTTTAACCCTGCTA3' and reverse primer sequence: 5'ATATTCTCGTGGCTTCCCAG3'. The PCR reaction was performed using a $25 \mu \mathrm{l}$ mixture as described by Lilakos et al., (2006). A 360-bp fragment was visualized on a $3 \%$ agarose gel stained with ethidium bromide under UV light. For analyzing the presence of type A mutation in NPM1 exon 12, we used a forward primer sequence: 5'CCAAGAGGCTATTCAAGATCTCTCTC3' and reverse primer sequence: 5'ACCATTTCCATGT CTGAGCACC3' according to Ottone et al., (2008). A 320-bp fragment was visualized by electrophoresis on $2 \%$ agarose gel. As internal control, we used ABL amplification with the following primers sequence: 5'GCATCTGACTTTGAGCCTCAG3' and 5'TGACTGGCGTGATGTAGTTGCTT3' with same PCR conditions and $258 \mathrm{bp}$ fragment visualized by electrophoresis on $2 \%$ agarose gel.

\section{Quantitative assessment of MDR1 gene expression}

$M D R 1$ gene expression was tested by real time PCR based on SYBR Green I fluorescence using StepOne (Applied Biosystems, USA). We used the following primer sequences for $M D R 1$ gene: F:5'-GCCAAAGCCAAAATATCAGC-3'and R: 5'-TTCCAATGTGTT CGGCAT-3'and GAPDH as the house keeping gene:F:5'-GAAGGTGAAGGTCGGAGT-3' and R:5'-GAAGATGGTGATGGGATTT C-3' as described by Yang et al. 2012. The relative quantification (RQ) of $M D R 1$ gene expression was assessed by $2^{-\Delta \Delta \mathrm{Ct}}$ method $(\Delta \Delta \mathrm{Ct}=\{[\mathrm{Ct}(\mathrm{MDR} 1 \mathrm{sample})-\mathrm{Ct}(\mathrm{GAPDH}$ sample $)]-$ 
[Ct(MDR1 control) - Ct (GAPDH control) $]\}$.

\section{Data analysis}

Statistical analysis was done using IBM $^{\odot}$ SPSS $^{\odot}$ Statistics version 22 (IBM ${ }^{\odot}$ Corp., Armonk, NY, USA). Numerical data were expressed as mean and standard deviation or median and range as appropriate. Qualitative data were expressed as frequency and percentage. Chi-square test or Fisher's exact test was used to examine the relation between qualitative variables. For not normally distributed quantitative data, comparison between two groups was done using Mann-Whitney test (non-parametric t-test). Spearman-rho method was used to test correlation between numerical variables. Survival analysis was done using Kaplan-Meier method and comparison between two survival curves was done using log-rank test. All tests were two-tailed. A p-value $<0.05$ was considered significant.

\section{Results}

\section{Patients' characteristics}

Our patients were 47 males and 53 females with a male to female ratio 0.9 . Their ages ranged between 5 and 70 years with a mean value of $38.7 \pm 15.9$ years. AML cases were stratified according to their age into adulthood cases $(93 \%)$ and childhood cases (7\%). Seventy one patients were de novo AML cases while 29 patients were in relapse. As regards FAB classification, 37\% were M1, $28 \%$ were $\mathrm{M} 2,15 \%$ were M4, $17 \%$ were M5 and $3 \%$ were M6. Molecular studies revealed 36 patients had FLT3/ ITD mutation and 41 patients had NPM1 mutation A. Regarding cytogenetic studies 35 patients were $\mathrm{t}(8 ; 21)$ positive and 27 patients were inv. 16 positive.

\section{MDR1 gene expression levels}

In the control group, MDR 1 gene expression level ranged between 0.53 and 2.6 with a mean value of $1.129 \pm 0.620$ and a median value of 0.894 . However, in AML patients, it ranged between 0.001 and 191.34 with a mean value of $8.672 \pm 26.260$ and a median value of 1.258 , with no statistically significant difference between the two groups $(\mathrm{P}=0.57)$. MDR 1 gene expression level in de novo patients ranged between 0.005 and 29.446 with a mean value of $3.637 \pm 6.5$ while in relapsed patient, it ranged between 0.132 and 191.34 with a mean value of $13.363 \pm 39.21$ and no statistically significant difference between the two groups $(\mathrm{P}=0.66)$. AML patients with $M D R 1$ gene expression level below cut off value which
Table 1a. Comparison between AML Patients with MDR1 Negative or Positive According to Their Clinical and Laboratory Data

\begin{tabular}{|c|c|c|c|}
\hline Parameter & $\begin{array}{c}\text { MDR1 negative } \\
\mathrm{n}=48(48 \%)\end{array}$ & $\begin{array}{c}\text { MDR1 positive } \\
n=52(52 \%)\end{array}$ & P-value \\
\hline \multicolumn{4}{|l|}{ Age } \\
\hline Adulthood & $44(47.3 \%)$ & $49(52.7 \%)$ & 0.71 \\
\hline Childhood & $4(57.1 \%)$ & $3(42.9 \%)$ & \\
\hline \multicolumn{4}{|l|}{ Gender } \\
\hline Male & $24(51.1 \%)$ & $23(48.9 \%)$ & 0.56 \\
\hline Female & $24(45.3 \%)$ & $29(54.7 \%)$ & \\
\hline \multicolumn{4}{|l|}{ FAB Classification } \\
\hline M1 & $19(51.4 \%)$ & $18(48.6 \%)$ & 0.132 \\
\hline M2 & $15(53.6 \%)$ & $13(46.4 \%)$ & \\
\hline M4 & $9(60 \%)$ & $6(40 \%)$ & \\
\hline M5 and M6 & $5(25 \%)$ & $15(75.8 \%)$ & \\
\hline \multicolumn{4}{|l|}{ Mol. studies } \\
\hline FLT3-ITD wild & $34(53.1 \%)$ & $30(46.9 \%)$ & 0.171 \\
\hline FLT3-ITD mutant & $14(38.9 \%)$ & $22(61.1 \%)$ & \\
\hline NPM1 WILD & $28(47.5 \%)$ & $31(42.9 \%)$ & 0.89 \\
\hline NPM1 mutant & $20(48.8 \%)$ & $21(51.2 \%)$ & \\
\hline \multicolumn{4}{|l|}{ Cytogenetic studies } \\
\hline$t(8 ; 21)$ positive & $16(45.7 \%)$ & $19(54.3 \%)$ & 0.74 \\
\hline$t(8 ; 21)$ negative & $32(49.2 \%)$ & $33(50.8 \%)$ & \\
\hline inv.16 positive & $12(44.4 \%)$ & $15(55.6 \%)$ & 0.67 \\
\hline inv.16 negative & $36(49.3 \%)$ & $37(50.7 \%)$ & \\
\hline
\end{tabular}

was the mean expression level in the control group+2SD (2.403) were considered as "MDR1 Negative", while those with expression level higher than (2.403) were considered as "MDR1 Positive". Forty eight patients were $M D R 1$ negative with MDR1 gene expression level ranged between 0.001 and 1.057, a mean value of $0.344 \pm 0.329$ and median value of 0.215 . Fifty two patients were MDR1 positive where $M D R 1$ gene expression level ranged between 1.133 and 191.34 with a mean value of $16.360 \pm 34.827$ and median value of 4.69. Statistical analysis showed significant difference in expression levels between the two groups with $\mathrm{p}$ value $=0.00$. Comparison between AML patients with MDR1 negative versus positive according to their clinical and laboratory data was described in Table 1a and $1 \mathrm{~b}$.

Correlation between MDR1 expression levels, FIT3/ITD and NPM1 mut. A and response to therapy

Complete remission (CR) was defined as recovery

Table 1b. Comparison between AML Patients with MDR1 Negative or Positive According to Their Laboratory Data

\begin{tabular}{lccccccc}
\hline Parameter & \multicolumn{2}{c}{ MDR1 negative $\mathrm{n}=48(48 \%)$} & \multicolumn{3}{c}{ MDR1 positive $\mathrm{n}=52(52 \%)$} & P-value \\
& Range & Mean \pm SD & Median & Range & Mean \pm SD & Median & \\
\hline $\mathrm{Hb} \mathrm{gm} / \mathrm{dL}$ & $3-13.7$ & $7.8 \pm 2.1$ & 7.6 & $3.7-12$ & $7.4 \pm 1.9$ & 7.3 & 0.343 \\
$\mathrm{TLCx} 10^{3} / \mathrm{cm}^{3}$ & $3.3-272$ & $55.625 \pm 62.1$ & 39 & $1.5-312$ & $62.996 \pm 67.7$ & 51.75 & 0.456 \\
$\mathrm{Plts} \times 10^{3} / \mathrm{cm}^{3}$ & $6-386$ & $80.8 \pm 87.1$ & 44.5 & $5-228$ & $48.7 \pm 53.7$ & 28 & $0.030^{*}$ \\
P.B blast (\%) & $10-95$ & $51.8 \pm 25.5$ & 52.5 & Oct-99 & $53.8 \pm 28.6$ & 55.5 & 0.702 \\
B.M blast (\%) & $21-99$ & $76.0 \pm 21.9$ & 86.5 & $21-99$ & $74.9 \pm 25.3$ & 85 & 0.836 \\
\hline
\end{tabular}




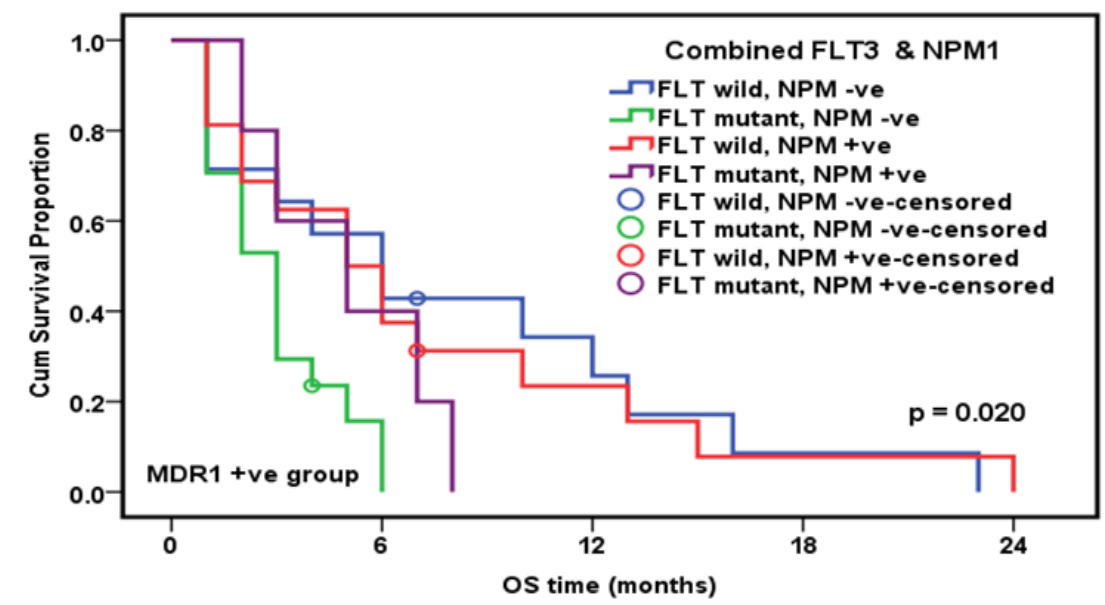

Figure 1. OS in MDR1 +ve Group Regarding FLT3/ITD and NPM1mut.A status

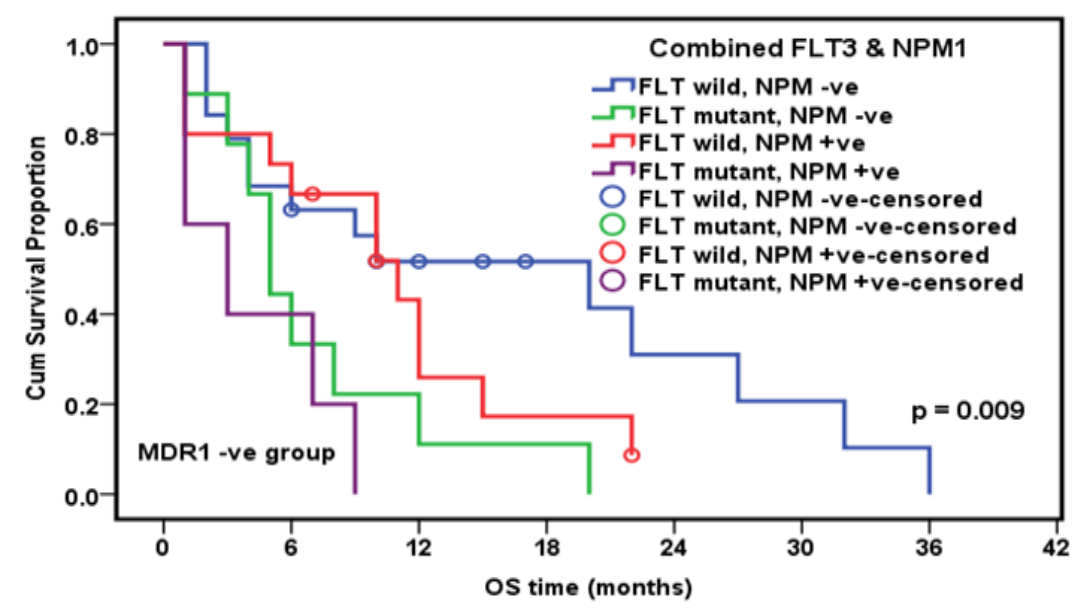

Figure 2. OS in MDR1 -ve Group Regarding FLT3/ITD and NPM1mut.A Status

of bone marrow morphology with less than $5 \%$ blasts, neutrophil count $1 / 109 / \mathrm{L}$ or more, platelet count 100/109/L or more, and no evidence of extra medullary leukemia. Resistant disease (RD) was defined as treatment resistance when evaluation did not meet the criteria of complete remission. Early death was defined as death before completion of the induction therapy cycle. These latter patients were not included in evaluation of resistant disease. Fifty seven patient achieved CR with MDRl gene expression level ranged between 0.003 and 29.45, with a mean value of $2.912 \pm 5.592$ and median value of 0.86 . Forty three patients had $R D$ with $M D R 1$ gene expression level ranged between 0.001 and 191.341 , with a mean value of $16.308 \pm 38.458$ and median value of 3.68 . There was statistically significant difference noticed in MDR1 gene expression between the two patients groups with $\mathrm{P}$ value $<0.001$. In this study, 48 patients were MDR1 negative with 32 patients achieved CR after induction therapy and 16 patients had RD. However, 52 patients were MDR1 positive with 25 patients achieved CR after induction therapy and 27 patients had RD. There was no statistically significant difference between patients as regards the response to therapy in the 2 groups ( $P$ value 0.061). Regarding FLT3/ITD mutation, there was statistically significant difference between cases who achieved CR and those with RD with p-value (0.002), where CR rates were higher in patients with wild FLT3/ ITD than in mutant FLT3/ITD patients. While NPM1 mutation A showed no statistically significant difference between cases who achieved CR and those with RD with $\mathrm{P}$-value 0.14. AML patients were stratified into groups according to MDR1 gene expression, FLT3-ITDs and NPM1 mutation A status. Statistical analysis of studied molecular genetic abnormalities regarding the response to therapy show significant difference between cases who achieved $\mathrm{CR}$ and those with $\mathrm{RD}$ within patients with MDR1 +ve with $\mathrm{P}$-value $=0.020 *$ where $\mathrm{CR}$ rate was higher with (FLT3/ITD wild, NPM1 mut.A neg.) and

Table 2. Correlation between MDR1 Expression Levels, FIT3/ITD \& NPM1 Mut. A and Response to Therapy

\begin{tabular}{llccc}
\hline & Subgroup & CR & RD & P-value \\
\hline & FLT3/ITD mutant, NPM1 +ve & 2 & 3 & \\
& FLT3/ITD mutant, NPM1 -ve & 3 & 14 & \\
MDR1 & FLT3/ITD wild, NPM1 -ve & 9 & 5 & $0.020^{*}$ \\
Positive & FLT3/ITD wild, NPM1 +ve & 10 & 6 & \\
& FLT3ITD mutant, NPM1 +ve & 3 & 2 & \\
& FLT3/ITD mutant, NPM1 -ve & 5 & 4 & \\
MDR1 & FLT3/ITD wild, NPM1 -ve & 13 & 6 & 0.349 \\
Negative & FLT3/ITD wild, NPM1 +ve & 12 & 3 & \\
\hline
\end{tabular}

*, Significant at $P \leq 0.05$ 


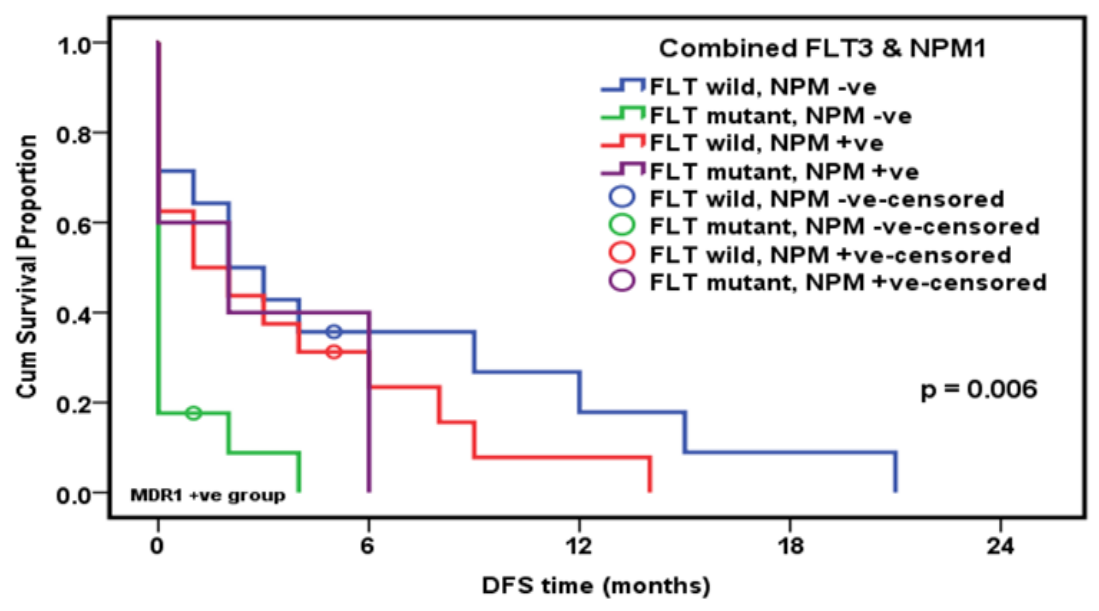

Figure 3. DFS in MDR1 +ve Group Regarding FLT3/ITD and NPM1mut.A Status

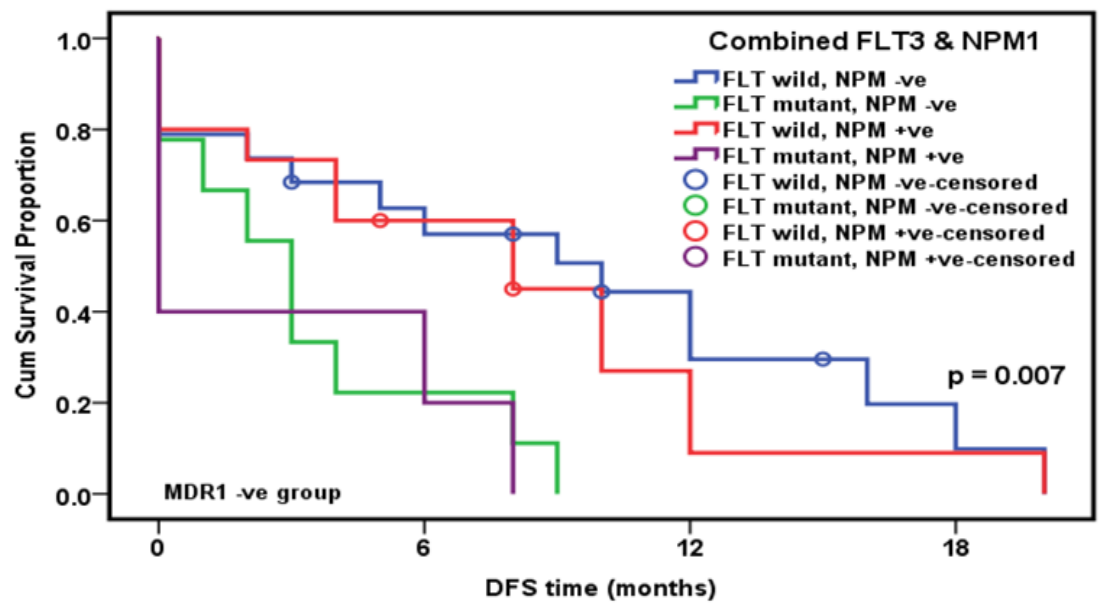

Figure 4. DFS in MDR1 -ve Group Regarding FLT3/ITD and NPM1mut.A Status

(FLT3/ITD wild, NPM1 mut.A pos.). While AML patients with MDR1 -ve showing no statistically significant difference between cases who achieved $\mathrm{CR}$ and those with $\mathrm{RD}$ with $\mathrm{P}$ value $=0.349$, Table 2 .

Correlation between MDR1 expression levels, FIT3/ITD and NPM1 mut. A and OS and DFS

AML patients were followed up for 6-24 months according to available clinical data, the Overall Survival rate (OS: defined from the date of diagnosis till the date the patient died, or was last seen) and the Disease Free Survival rate (DFS: defined from the date of CR achievement till the date the patient relapsed) were assessed. AML patients with MDR1 negative had a cumulative DFS at 6 months $47.3 \%$, while patients with MDR1 positive had a cumulative DFS at 6 months of $16.9 \%$, with statistically significant difference encountered between the two patients' group ( $\mathrm{P}$ value 0.004$)$. While, AML patients with MDR1 negative had a cumulative OS at 6 months $56.3 \%$, while patients with MDR1 positive had a cumulative OS at 6 months $28 \%$, with statistically significant difference between the two patients ' group $(\mathrm{P}$ value 0.01 ). After stratifying AML patients into groups according to studied molecular genetic abnormalities. MDR1 +ve group and MDR1 -ve showing significant higher OS and DFS in FLT3/ITD wild patients whether
NPM1 is mutant or not, Figures 1-4.

\section{Discussion}

Multidrug resistance may be an inherent phenomenon seen before medications or acquired after an initially successful treatment begins (Filipits, 2004). The MDR phenotype is described as the simultaneous resistance against various drugs that have no structural similarity and act on different molecular targets. It is a multifactorial phenomenon with biochemical resistance mechanisms in common, which can include the reduction of intracellular drug concentrations by changes in its influx/efflux (Larsen et al., 2000). Here, we aim to investigate the expression of the MDR1 gene in AML patients, to identify their role on both the progression and chemotherapeutic refractoriness. As this is important chemotherapeutic drug transporters, quantification of this gene by real-time PCR offers particular promise as prognostic markers and markers for drug resistance in AML patients together with assessment of known prognostic molecular markers; FLT3-ITD and NPM1 mutations. In our study, MDR1 gene expression was insignificantly higher in AML cases compared to controls with $\mathrm{P}$ value $=0.57$. This is in agreement with (Xu et al., 2008; Farawela et al., 2014) who reported that the expression of $M D R 1$ gene was significantly higher in 
AML patients than in normal controls. Also, MDR1 gene expression was insignificantly higher in relapsed cases compared to de novo cases with $\mathrm{P}$ value $=0.66$. Several studies have reported a higher MDR1 expression at time of relapse as compared to time of diagnosis (Farawela et al., 2014; Beck et al, 1996; Gekeler et al., 1992). However, most studies suggest an identical expression or even lower level of MDR 1 in relapsed/refractory AML (Hart et al., 1994; Sato et al., 1990). Moreover, Huh et al., 2006 reported that MDR mRNA expression did not give significant effects on tendency to relapse or continuous CR status after induction chemotherapy. Our AML patients were divided into 2 groups; MDR1 negative and MDR1 positive according to Farawela et al., 2014. Forty eight patients were MDR1-negative and 52 patients were MDR1positive. This is in accordance with (Leith et al., 1999; Fujimaki et al., 2002). Their studies reported that over a third of AML patients had MDR1 expression. Also, these frequencies were higher than those previously reported in the study of Schaich et al., (2004) who reported that $25 \%$ their AML patients had MDR1expression-positive in addition to (Huh et al., 2006; Farawela et al., 2014) who reported that MDR1 gene was expressed in 23.1 $\%$ and $39.6 \%$ of AML patients, respectively. On the contrary, Pirker et al., (1991) reported positive MDR1 gene expression in about $71 \%$ of their AML cases. There was no statistically significant difference noticed between AML patients with MDR1-negative and MDR1-positive as regards their age, gender, FAB classification or other clinical and laboratory features except for platelet count which was higher in MDR1 negative patients. This is in accordance with (Illmer et al., 2002; Farawela et al., 2014). As regards response to induction therapy; $M D R 1$ gene expression levels were significantly higher in AML patients with $\mathrm{RD}$ with $\mathrm{P}$ value $<0.001$. Our results are similar to Farawela et al., 2014 who observed that MDR1 gene expression levels were higher in cases with RD than cases who achieved CR; however it didn't reach statistically significant difference. FLT3ITD represents one of the most frequent genetic alterations in AML. In our study FLT3/ITD showed statistically significant difference between cases who achieved CR and those with resistant disease were FLT3/ITD higher in patients with RD. This is in agreement with Wang et al., (2017) who reported that FLT3ITD-positiveAML patients had higher relapse incidence. These observations have validated FLT3 as a therapeutic target in AML with FLT3 inhibitors have shown promising results when combined with standard therapy. In our study, NPM1 showed statistically insignificant difference between cases who achieved CR and those with RD were NPM1 mutation was higher in patients with CR. Similarly, Zidan et al., (2013) reported that NPM1 mutation is a prognostic factor for a favorable outcome in Egyptian population. The current work aimed to verify the impact of the studied molecular genetic abnormalities on the response to therapy. AML patients were stratified into groups according to $M D R 1$ gene expression, FLT3/ITDs and NPM1 mutation A status. In MDR positive group, wild FLT3/ITD with or without NPM1 mutation was favorable in achieving CR with $p$ value (0.02). In MDR negative group, wild FLT3/ITD with or without NPM1 mutation showed insignificantly higher $\mathrm{CR}$ rates with $\mathrm{p}$ value (0.35). However, Farawela et al., (2014) reported that there was no statistically significant difference noticed between cases who achieved CR and those with RD except for those with negative for MDR1 expression together with mutated NPM gene where CR rate was higher in this group. Our patient with wild FLT3/ ITD showed significantly higher DFS and OS with $\mathrm{p}$ value 0.001 and $<0.001$, respectively. Similar to Wang et al., (2017) who reported that FLT3ITD-positive AML patients had lower DFS as well as OS. Our Patient with NPM mutant showed insignificantly higher DFS and OS with $p$ value 0.709 and 0.708 , respectively. This is in agreement with that previously reported by Verhaak et al., (2005) who reported that NPM1 mutations express independent favorable prognostic value with regard to OS, and DFS. Kaplan-Meier curves revealed statistically significant difference between MDR1-negative and MDR1-positive patients regarding their DFS and OS between the two groups where DFS and OS were higher in MDR1-negative patients with $p$ value 0.004 and 0.01 , respectively. This is compatible to that previously reported by (Pirker et al. 1991; Schaich et al., 2004) who reported a significant decrease in both DFS and OS of AML patients with detectable $M D R 1$ gene expression compared to the DFS and OS of MDR1 RNA-negative patients. Also, Huh et al., 2006 reported that MDR1-positive AML had poor 2 -yr survivals; yet this observation was not supported by statistical analysis.

In conclusion, the results obtained by the current work together with the previous researches concerning the study of multidrug resistance genes in acute myeloid leukemia patients providing additional evidence of the role played by these genes as predictors of chemoresistance and poor treatment outcome. Hence, MDR1 blockade-mediated chemoresistance reversal, or novel chemotherapeutic approaches directed against cancer expressing MDR1 could enhance tumor eradication and contribute to durable clinical response.

\section{Acknowledgements}

We deeply acknowledge the scientific and technical support offered by Oncology dep. molecular lab team. We are also thankful for our dear patients as well as IT dep. team.

\section{Conflicts of interest}

Authors declared no conflicts of interest.

\section{Ethical approval}

All procedures performed in the study involving human participants were in accordance with the ethical standards of the institutional research committee and with the 1964 Helsinki declaration and its later amendments (GCP guidelines) or comparable ethical standards.

Informed consent was obtained from all individual participants included in the study. 


\section{References}

Almeida AM and Ramos F (2016) Acute myeloid leukemia in the older adults. Leuk Res Rep, 6, 1-7.

Beck WT, Grogan TM, Willman CL (1996) Methods to detect P-glycoprotein-associated multidrug resistance in patients' tumors: consensus recommendations. Cancer Res, 56, 3010-20.

Daver N, Schlenk RF, Russell NH, Levis MJ (2019) Targeting FLT3 mutations in AML: review of current knowledge and evidence. Leukemia, 33, 299-12.

Döhner H, Weisdorf DJ, Bloomfield CD (2015) Acute myeloid leukaemia. $N$ Engl J Med, 373, 1136-52.

Farawela HM, Khorshied MM, Kassem NM, Kassem HA, Zawam HM (2014) The clinical relevance and prognostic significance of adenosine triphosphate ATP-binding cassette (ABCB5) and multidrug resistance (MDR1) genes expression in acute leukemia: an Egyptian study. J Cancer Res Clin Oncol, 140, 1323-30.

Filipits M (2004) Mechanism of cancer: Multidrug resistance. Drug Discov Today, 1, 229-4.

Fujimaki S, Funato T, Harigae H, et al (2002) Quantitative analysis of a MDR1 transcript for prediction of drug resistance in acute leukemia. Clin Chem, 48, 811-7.

Gekeler V, Frese G, Noller A (1992) Mdr1/P-glycoprotein, topoisomerase, and glutathione-S-transferase $p i$ gene expression in primary and relapsed state adult and childhood leukaemias. Br J Cancer, 66, 507-17.

Grafone T, Palmisano M, Nicci C, Storti S (2012) An overview on the role of FLT3-tyrosine kinase receptor in acute myeloid leukemia: biology and treatment. Oncol Rev, 6, 64-74.

Hart SM, Ganeshaguru K, Hoffbrand AV, Prentice HG, Mehta AB (1994) Expression of the multidrug resistance- associated protein (MRP) in acute leukaemia. Leukemi, 8, 2163-8.

Hatakeyama H, Harashima H (2014) Cancer multidrug resistance: mechanisms involved and strategies for circumvention using a drug delivery system. Arch Pharm Res, 37, 4-15.

Huh HJ, Park CJ, Jang S, et al (2006). Prognostic significance of multidrug resistance gene 1 (MDR1), multidrug resistancerelated protein (MRP) and lung resistance protein (LRP) mRNA expression in acute leukemia. J Korean Med Sci, 21, 253-8.

Illmer T, Schuler US, Thiede C, et al (2002). MDRl Gene Polymorphisms affect therapy outcome in acute myeloid leukemia patients. Cancer Res, 62, 4955-2.

Larsen AK, Escargueil AE, Skladanowski A (2000) Resistance mechanisms associated with altered intracellular distribution of anticancer agents. Pharmacol Ther, 85, 217-29.

Leith CP, Kopecky KJ, Chen IM, et al (1999). Frequency and clinical significance of the expression of the multidrug resistance proteins MDR1/P-glycoprotein, MRP1, and LRP in acute myeloid leukemia. A southwest oncology group study. Blood, 94, 1086-99.

Leung AY, Man CH, Kwong YL (2013). FLT3 inhibition: a moving and evolving target in acute myeloid leukaemia. Leukemia, 27, 260-8

Lilakos K, Viniou NA, Mavrogianni D, et al (2006). FLT3 Overexpression in acute promyelocytic leukemia patients without detectable FLT3-ITD or codon 835-836 mutations: A pilot study. Anticancer Res, 26, 1201-8.

Liu H, Tan BCM, Tseng KH, et al (2007). Nucleophosmin acts as a novel AP2 $\alpha$-binding transcriptional corepressor during cell differentiation. EMBO Rep, 8, 394-400.

Livak KJ, Schmittgen TD (2001). Analysis of relative gene expression data using real-time quantitative PCR and the 2(-delta delta C (T)) method. Methods, 25, 402-8.

Ottone T, Ammatuna E, Lavorgna S, et al (2008). An allele-specific RT-PCR assay to detect type A mutation of the nucleophosmin-1 gene in acute myeloid leukemia. $J M D, 10,212-6$.

Pirker R, Wallner J, Geissler K, et al (1991). MDRl gene expression and treatment outcome in acute myeloid leukemia. J Natl Cancer Inst, 83, 708-12.

Sato H, Preisler H, Day R (1990). MDR1 transcript levels as an indication of resistant disease in acute myelogenous leukaemia. Br J Haematol, 75, 340-5.

Schaich M, Soucek S, Thiede C, Ehninger G, Illmer T (2004) $M D R 1$ and $M R P 1$ gene expression are independent predictors for treatment outcome in adult acute myeloid leukaemia. Br J Haematol, 128, 324-32.

Scheffer GL, Wijngaard PL, Flens MJ, et al (1995). The drug resistance related protein LRP is the human major vault protein. Nat Med, 1, 578-2.

Styczynski J (2007). Drug resistance in childhood acute myeloid leukemia. Curr Pharm Biotechnol, 8, 59-75.

Suleiman AA, Al-Saffar AZ, Rafaa TA, Jwad HA (2018). Genetic mutation assessment of NPM1 gene and gene expression of $M D R 1$ in Iraqi patients with acute myeloid leukemia. OnLine $J$ Biol Sci, 18, 37-5.

Takahashi S (2011). Downstream molecular pathways of FLT3 in the pathogenesis of acute myeloid leukemia: biology and therapeutic implications. J Hematol Oncol, 4, 13-23

Tiribelli M, Geromin A, Michelutti A, et al (2011). Concomitant ABCG2 overexpression and FLT3-ITD mutation identify a subset of acute myeloid leukemia patients at high risk of relapse. Cancer, 117, 2156-2.

Verhaak RG, Goudswaard CS, van Putten W, et al (2005). Mutations in nucleophosmin NPM1 in acute myeloid leukemia (AML): association with other gene abnormalities and previously established gene expression signatures and their favorable prognostic significance. Blood, 106, 3747-4.

Wang M, Yang C, Zhang L, Schaar DG (2017). Molecular mutations and their cooccurrences in cytogenetically normal acute myeloid leukemia. Stem Cells Int, 2017, 1-11.

Wei AH, Tiong IS (2017) Midostaurin, enasidenib, CPX-351, gemtuzumab ozogamicin, and venetoclax bring new hope to AML. Blood, 130, 2469-4.

Xu B, Song XY, Li L, Xu WJ, Tang JH (2008) The significance of quantification of $M D R 1$ and $W T 1$ gene expression in acute myeloid leukemia. Zhonghua Nei Ke Za Zhi, 47, 221-4.

Yang M, Li W, Fan D, et al (2012). Expression of ABCB5 gene in hematological malignances and its significance. Leuk Lymphoma, 53, 1211-5.

Zidan M, Shaaban H, El Ghannam D (2013). Prognostic impact of nucleophosmin 1 (NPM1) gene mutations in Egyptian acute myeloid leukemia patients. Turk J Haematol, 30, 129-6.

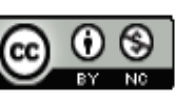

This work is licensed under a Creative Commons AttributionNon Commercial 4.0 International License. 